\title{
ANÁLISE COMPARATIVA DE CONTROLADORES REPETITIVOS MODIFICADOS PARA APLICAÇÃO EM INVERSORES PWM
}

\author{
Leandro Michels* \\ michels@gepoc.ufsm.br \\ Márcio Stefanello ${ }^{\dagger}$ \\ marciostefanello@unipampa.edu.br \\ Hilton Abílio Gründling* \\ ghilton03@gmail.com \\ *Universidade Federal de Santa Maria \\ Grupo de Eletrônica de Potência e Controle \\ Av. Roraima 1000, Campus Universitário, Camobi, CEP 97105-900 \\ Santa Maria, RS, Brasil \\ ${ }^{\dagger}$ Universidade Federal do Pampa / Campus Alegrete \\ Centro de Tecnologia \\ Av. Tiarajú 810, Bairro Ibirapuitã, CEP 97546-550 \\ Alegrete, RS, Brasil
}

\section{ABSTRACT}

Comparative Analysis of Modified Repetitive Controllers for Applications in PWM Inverters

Conventional repetitive controllers are not suitable for applications were reference or disturbances are periodic where its fundamental frequency is subject to variation. However, this is a situation commonly encountered in applications of pulse-width modulated inverters feeding nonlinear loads or connected to the grid. Several approaches to mitigate this problem have been proposed in the literature, but there is no studies with comparative analysis among them. This paper provides a comparative analysis of three modified repetitive controllers developed to solve this problem. The following methods are covered in the analysis: i) continuous adjustment of the sampling rate; ii) truncation of the number of samples per reference cycle to the nearest integer and iii) adjustment of a fictitious sampling rate in order to maintain constant the number of samples of the controller per reference cycle. These existing approaches are depicted in details

Artigo submetido em 07/02/2011 (Id.: 1263)

Revisado em 16/03/2011, 24/08/2011

Aceito sob recomendação do Editor Associado Prof. Francisco de Assis dos Santos Neves
\end{abstract}

and its performances are discussed based on the experimental results obtained in two experimental prototypes, which had been carefully designed to operate at low and high switching frequencies.

KEYWORDS: Repetitive control, digital control, PWM inverter, AC power source.

\section{RESUMO}

Controladores repetitivos convencionais não são adequados para aplicações onde os sinais de referência ou os distúrbios são periódicos mas cuja frequência fundamental pode sofrer modificações. Contudo, esta é uma situação frequentemente encontrada em aplicações de inversores de tensão modulados por largura de pulso alimentando cargas não-lineares ou conectados à rede elétrica. Várias propostas para a mitigação deste problema são encontradas na literatura especializada, mas não se reporta estudos analisando-as comparativamente. Neste sentido, este trabalho apresenta uma análise comparativa de três controladores repetitivos modificados desenvolvidos para resolver este problema. São considerados os seguintes métodos: i) ajuste contínuo da taxa de amostragem, 
ii) truncamento do número de amostras por ciclo da referência para o número inteiro mais próximo e iii) ajuste de uma taxa de amostragem fictícia de modo a manter constante o número de amostras do controlador por ciclo da referência. Estas abordagens são descritas em detalhes e os seus desempenhos são discutidos com base em resultados experimentais obtidos em dois protótipos distintos, que foram criteriosamente projetados para operar em altas e baixas frequências de comutação.

PALAVRAS-CHAVE: Controlador repetitivo, controle digital, inversor PWM, fonte de tensão CA de potência.

\section{INTRODUÇÃO}

Inversores de tensão com Modulação por Largura de Pulso (PWM, do inglês Pulse Width Modulation) são comumente utilizados em aplicações onde é necessário um elevado desempenho, tais como em Fontes Ininterruptas de Energia (UPS, do inglês Uninterruptible Power Supply), fontes de potência CA programáveis e filtros ativos de potência. Do ponto de vista de controle, a capacidade de rastreamento de referência e de rejeição de distúrbios, que são inerentes nestas aplicações, são requisitos imprescindíveis. Por estes motivos, a aplicação do chamado Controlador Repetitivo (CR) (Hara et al., 1988; Tomizuka et al., 1989) têm sido considerada como uma alternativa para a compensação de erros de regime permanente na maioria destas aplicações, tais como em UPSs (Michels et al., 2004; Escobar et al., 2007; Zhou et al., 2006), fontes CA (Tzou et al., 1997) e filtros ativos de potência conectados em paralelo na rede elétrica (Grino et al., 2007; Olm, Ramos, Castello-Castelló and Cardoner, 2010). O CR está atualmente, também sendo utilizado no controle de inversores para outras aplicações, tais como em geração distribuída (Mastromauro et al., 2009), correção de fator de potência (Wu et al., 2010) e fontes CC (Escobar et al., 2005).

O CR teve sua origem no Princípio do Modelo Interno (Francis and Wonham, 1975) e foi desenvolvido com o objetivo de minimizar o erro de regime permanente em aplicações onde o sinal de referência é periódico, e os distúrbios são variáveis de entrada exógenas e periódicas envolvendo componentes harmônicas de uma mesma frequência fundamental. Consequentemente, o CR é adequado para aplicações de inversores PWM, dado que os sinais de referência são geralmente senoidais ou então formados por uma soma de componentes harmônicas. Adicionalmente, nestas aplicações, o comportamento da carga não-linear é similar a um distúrbio exógeno sincronizado com o sinal de referência (Michels et al., 2004).

Existem vários tipos de CRs adequados para implementação digital (Tomizuka et al., 1989; Kempf et al., 1993). Para inversores PWM, o algoritmo do tipo filtro-Q é o esquema mais utilizado ser de rápida execução e velocidade convergência e possuir código de baixa complexidade. Entretanto, para se obter um bom desempenho, o CR com filtro-Q necessita dos seguintes requisitos (Tomizuka et al., 1989): i) O conhecimento do período da referência e do distúrbio e ii) um número fixo e inteiro de amostras do $\mathrm{CR}$ por período da referência. Este último requisito garante o "sincronismo entre os períodos" do controlador e da referência e/ou do distúrbio.

O uso de controladores repetitivos em inversores de tensão PWM têm sido tema de vários estudos no Brasil. Alguns destes trabalhos propuseram o uso de CRs em filtros ativos de potência, tal como em (Marafo et al., 2004) que empregao para a rejeição seletiva de harmônicas em um filtro paralelo, e (de Melo et al., 2010) para o controle de um filtro universal. Outros estudos foram conduzidos com foco em aplicações em UPS, com destaque para novas metodologias de projeto (Michels and Gründling, 2005; Montagner and Peres, 2007; Flores et al., 2010), algoritmo para aplicações em inversores com transformador de saída (Bottern and Pinheiro, 2005), algoritmo para aplicações com inversores intercalados (Contreras et al., 2010), técnicas de reinicialização dos CRs na ocorrência de distúrbios não periódicos (Rech et al., 2010), algoritmo adaptativo (Stefanello and Grndling, 2007), algoritmo modificado para operação com referências de período variável (Michels et al., 2009) e técnicas anti-windup (Michels et al., 2010).

O perfeito sincronismo entre o período do CR e da referência não é, em geral, obtido perfeitamente em UPSs de duplaconversão, em fontes de potência CA programáveis utilizadas em testes de conformidade e em filtros ativos. No primeiro caso, uma chave de transferência (bypass) é utilizada para alternar as fontes de alimentação da carga entre a UPS e a rede elétrica, cuja frequência não pode ser assumida com sendo fixa. Problema semelhante ocorre no caso de filtros ativos, uma vez que este faz parte de um sistema conectado na rede elétrica. Em fontes de potência CA para testes de conformidade, a referência pode ter sua frequência variada com o objetivo de ensaiar determinado equipamento. Logo, estes são alguns exemplos de aplicações onde não é possível garantir o sincronismo entre os períodos do CR e a referência ou o distúrbio.

Diversos CRs modificados têm sido desenvolvidos para operarem adequadamente com sinais de referência de frequência variável. Vários destes trabalhos estão relacionados com teoria de controle (Steinbuch, 2002; Gupta and Lee, 2006; Pipeleers et al., 2008; Olm, Ramos, and Costa-Castelló, 2010) e mecatrônica (Manayathara et al., 1996; Hillerstrom, 1996; Cao and Ledwich, 2002; Fujimoto, 2009). Com relação a aplicação de CRs em inversores PWM tem-se alguns trabalhos tais como os de (Tzou et al., 1997; Olm, Ramos, Costa- 
Castelló and Cardoner, 2010; Jung et al., 1998; ?; Wang et al., 2007; Michels et al., 2009; Hornik and Zong, 2010). As principais soluções propostas são as seguintes: i) a utilização do $\mathrm{CR}$ convencional com um identificador de período para ajustar a taxa de amostragem (Olm, Ramos, CostaCastelló and Cardoner, 2010); ii) truncamento do número de amostras em cada ciclo da referência para o número inteiro mais próximo (Tzou et al., 1997; Jung et al., 1998); iii) o ajuste da frequência para a transformação- $d q$ de modo a garantir um número fixo de amostras do controlador por ciclo da referência (?); iv) a utilização de um CR com atraso de transporte operando numa frequência de amostragem constante (Wang et al., 2007); v) a alteração do tamanho dos buffers circulares utilizados para implementar o CR (Michels et al., 2009) e vi) a alteração em tempo real da frequência de corte do filtro passa-baixa utilizado para implementar o bloco do modelo interno (Hornik and Zong, 2010).

Apesar de terem sido apresentadas várias propostas para a solução para o problema, os autores não reportaram estudos comparando-as. Com o objetivo de cobrir essa lacuna, a contribuição do presente trabalho é o provimento de uma análise comparativa do impacto de CRs alguns modificados aplicados no controle de uma UPS com chave de transferência sujeita à variação de frequência da referência. Devido a grande quantidade de técnicas propostas na literatura optou-se por comparar apenas três destas técnicas. Escolheu-se as seguintes técnicas: i) ajuste da taxa de amostragem (Mattavelli and Marafao, 2004; Olm, Ramos, Costa-Castelló and Cardoner, 2010), por ser a técnica mais usual; ii) a alteração do tamanho dos buffers circulares utilizados para implementar o CR (Michels et al., 2009), por apresentar um algoritmo de implementação muito simples; iii) ajuste da taxa de amostragem fictícia (Cao and Ledwich, 2002), por ser a técnica de uso genérico mais consolidada.

A comparação foi relizada experimentalmente empregando um inversor PWM de $1 \mathrm{kVA}$. Dois protótipos distintos foram implementados de modo a emular aplicações de inversores tanto com alta quanto com baixa potência. Para tanto, os inversores dos protótipos foram configurados para operarem com baixa e elevada frequências de comutação, respectivamente. Em ambos os casos, as frequências de comutação e de amostragem são iguais e sincronizadas entre si.

Este artigo é organizado como segue: A Seção 2 descreve o modelo da planta e a estrutura do controlador. A Seção 3 descreve os CRs considerados para a análise e a Seção 4 apresenta a análise comparativa baseada nos resultados experimentais. A Seção 5 conclui o trabalho.

\section{DESCRIÇÃO DO SISTEMA}

O circuito do inversor PWM monofásico é mostrado na Fig. 1. Como mostrado, as cargas possuem em geral um retificador não-controlado no estágio de entrada, cujo comportamento é descrito pela Norma IEC 62040-3 (IEC 620403, 1999).

\subsection{Modelo da Planta}

O modelo dinâmico do inversor mostrado na Fig. 1 é nãolinear devido à carga retificada. De modo a linearizar este modelo, assume-se que a corrente de carga $i_{o}$ é um distúrbio exógeno (Escobar et al., 2007; Zhou et al., 2006). As seguintes hipóteses são feitas: i) o inversor opera em modo de condução contínua; ii) a frequência de comutação é muito maior que a máxima frequência da lei de controle $u$; iii) as harmônicas na tensão de saída $v_{o}$ devido a modulação são fortemente atenuadas. Uma vez que a forma de onda PWM $v_{a b}(t)$ é utilizada para sintetizar a ação de controle $u(t)\left(v_{a b}(t) \simeq u(t)\right)$, o sistema pode ser representado pelo seguinte sistema linear e invariante no tempo:

$$
\begin{gathered}
V_{o}(s)=G_{p}(s) V_{a b}(s)+G_{d}(s) I_{o}(s) \\
G_{p}(s)=\frac{V_{o}(s)}{V_{a b}(s)}=\frac{s C r_{C}+1}{s^{2} L C+s C\left(r_{L}+r_{C}\right)+1} \\
G_{d}(s)=\frac{V_{o}(s)}{I_{o}(s)}=\frac{s\left(C r_{C}^{2}+L\right)+r_{L}-r_{C}}{s^{2} L C+s C\left(r_{L}+r_{C}\right)+1}
\end{gathered}
$$

onde $\cdot(s)$ denota a Transformada de Laplace da função do domínio do tempo $\cdot(t)$, e $r_{C}$ and $r_{L}$ são, respectivamente, as resistências série equivalente do capacitor $C$ e do indutor $L$ (ver Fig. 1).

Para obter as funções de transferência de (2) e (3), assumese que: i) a ação de controle $v_{a b}(t)$ é gerada digitalmente

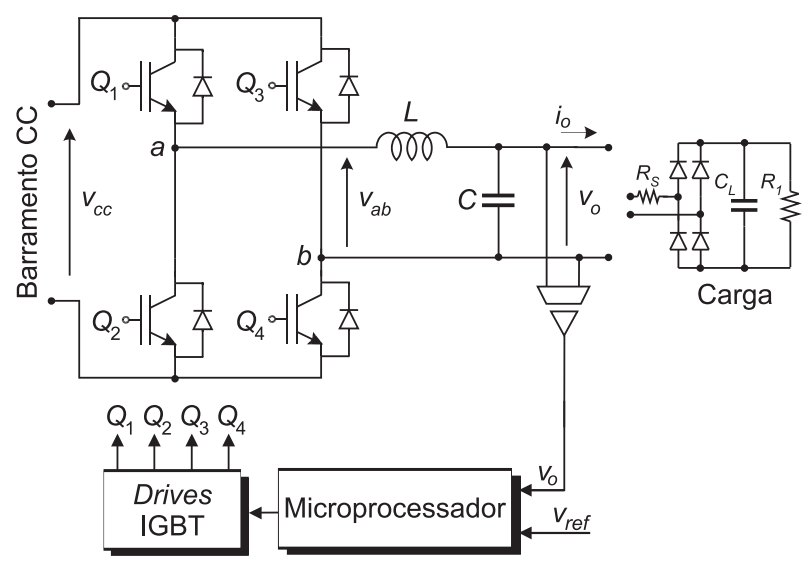

Fig. 1: Inversor PWM monofásico com carga não-linear. 


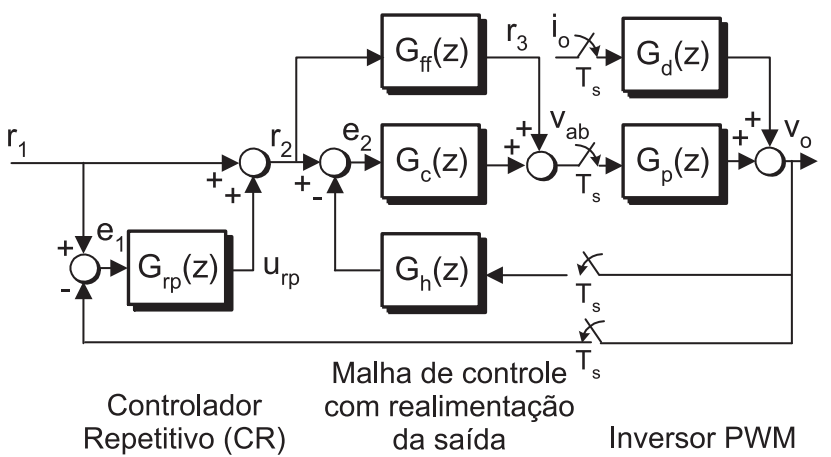

Fig. 2: Sistema de controle de tempo discreto.

por um modulador de largura de pulso, cujo comportamento é semelhante ao de um amostrador-retentor de ordem zero $(\mathrm{ZOH})$; ii) o distúrbio de entrada $i_{o}(t)$ pode ser representado pela sua aproximação $\mathrm{ZOH}$; iii) $v_{a b}(t), i_{o}(t)$ e $v_{o}(t)$ são amostradas simultaneamente numa frequência constante $f_{s}$. Como resultado, as funções de transferência de (2) e (3) no domínio do tempo discreto (domínio $z$ ) são dadas, respectivamente, por:

$$
\begin{aligned}
G_{p}(z) & =\frac{b_{1} z+b_{0}}{z^{2}+a_{1} z+a_{0}} \\
G_{d}(z) & =\frac{d_{1} z+d_{0}}{z^{2}+a_{1} z+a_{0}}
\end{aligned}
$$

onde $a_{0}, a_{1}, b_{0}, b_{1}, d_{0}$ e $d_{1}$ são constantes.

\subsection{Estrutura do Controlador}

A Fig. 2 descreve a estrutura do controlador do inversor PWM monofásico, incluindo as funções de transferência $G_{p}(s)$ e $G_{d}(s)$ da planta.

O esquema de controle mostrado na Fig.2 faz uso de uma malha de controle com realimentação da saída $v_{o}$ para compensação da resposta transitória. Um CR é empregado para a compensação de cargas não-lineares cíclicas, colaborando para o desempenho de regime permanente.

A estrutura do controlador da Fig.2 possibilita a análise de diferentes tipos de controladores tais como PID, deadbeat, por modelo de referência, alocação de pólos, etc., com ou sem ação de alimentação a frente (feedforward). A função de transferência de malha fechada envolvendo a saída $v_{o}$ e a referência $r_{2}$ é dada por:

$$
G_{m}(z)=\frac{V_{o}(z)}{R_{2}(z)}-\frac{G_{p}(z)\left[G_{f f}(z)+G_{c}(z)\right]}{1+G_{p}(z) G_{c}(z) G_{h}(z)}
$$

Neste artigo foi utilizado o controlador $G_{c}(z)=k_{1} z^{-1}+$ $k_{2} z^{-2}$ em conjunto com a ação feedforward $G_{f f}(z)=$
$G_{h}(z)=1$. Deste modo, a ação de controle é implementada utilizando a seguinte equação diferença ${ }^{1}$ :

$$
v_{a b}{ }^{*}(k+1)=k_{1} e_{2}(k)+k_{2} e_{2}(k-1)+r_{2}(k+1)
$$

onde $e_{2}=r_{2}-v_{o}$.

A função de transferência do bloco que implementa o CR com $n=f_{s} / f_{1}\left(n \in \mathbb{N}^{+}\right)$amostras num período do sinal de referência, é dada por:

$$
G_{r p}(z)=\frac{U_{r p}(z)}{E_{1}(z)}=\frac{1}{z^{n}-Q(z)} C(z)
$$

onde $Q(z)$ e $C(z)$ são filtros utilizados para aumentar a margem de estabilidade e compensar o atraso de fase de $G_{m}(z)$, respectivamente (Hara et al., 1988). Várias estruturas podem ser utilizadas para $Q(z)$ e $C(z)$ (Michels et al., 2004). De modo a simplificar a análise, neste artigo considerar-seá $Q(z)=q_{r}$ e $C(z)=c_{r} z^{d}$, onde $q_{r} \leq 1, c_{r}$ é o ganho da ação repetitiva e $z^{d}$ é o filtro utilizado para compensar o atraso de fase de $G_{m}(z)$. A ação repetitiva é implementada utilizando a seguinte equação diferença:

$$
u_{r p}(k+1)=q_{r} u_{r p}(k-n+1)+c_{r} e_{1}(k-n+d+1)
$$

onde $e_{1}=r_{1}-v_{o}$. Como resultado, a referência para a malha de controle de ação instantânea é dada por:

$$
r_{2}(k+1)=r_{1}(k+1)+u_{r p}(k+1)
$$

Neste artigo, a referência $r_{1}$ é uma forma de onda senoidal com frequência fundamental $f_{1}$.

\section{CONTROLADORES REPETITIVOS MO- DIFICADOS PARA RASTREAMENTO DE REFERÊNCIAS DE FREQUÊNCIA VARIÁVEL}

Controladores repetitivos com algoritmo do tipo filtro-Q reduzem significativamente a impedância de saída em regime permanente numa faixa estreita de frequências em torno das harmônicas múltiplas de $f_{s} / n$ (Michels et al., 2009). Portanto, uma elevada capacidade de rejeição de distúrbios é obtida apenas quando a seguinte condição é garantida:

$$
f_{1}=\frac{f_{s}}{n}
$$

No caso de referências de frequência variável, é necessário se identificar $f_{1}$ em tempo real. Desta forma, torna-se possível a adequação de $f_{s}, n$, ou ambos para satisfazer a relação dada em (11).

\footnotetext{
${ }^{1} \mathrm{~A}$ variável $k$ é o índice associado ao instante de tempo $t_{k}=k T_{s}$, $k=1,2, \ldots$, no qual uma variável $x(t)$ é amostrada resultando em $x(k)$. $T_{s}$ é o período de amostragem.
} 
Nesta seção inicialmente é apresentado o algoritmo utilizado para a determinação da frequência $f_{1}$. Este algoritmo é necessário para aplicações onde o sinal de referência não é gerado internamente pelo controlador, como nas aplicações em UPS e filtros ativos. Destaca-se que este algoritmo também pode ser empregado em aplicações com gerações interna de referência. Na sequência, os três CRs analisados neste artigo são apresentados. Estes algoritmos são formulados com o objetivo de satisfazer a condição apresentada em (11).

Os controladores repetitivos apresentados neste artigo consideram a aplicação em UPSs e em fontes CA programáveis para testes de conformidade. Nestas aplicações, as seguintes hipóteses são assumidas para o sinal de referência: i) $r_{1}$ é uma forma de onda senoidal cuja frequência fundamental $f_{1}{ }^{*}$ é conhecida; ii) $r_{1}$ não possui alterações súbitas de fase; iii) $r_{1}$ é livre de ruídos devido à característica passabaixa do algoritmo de sincronização (utilizado nas aplicações em UPS); iv) a faixa de valores de $f_{1}$ é conhecida $\left(f_{1_{\min }}<f_{1}<f_{1_{\max }}\right)$ e v) $f_{1}$ não varia mais que $\pm 1 \mathrm{~Hz} / \mathrm{s}$.

\subsection{Determinação de $f_{1}$}

O algoritmo utilizado para determinar $f_{1}$ é baseado num detector de passagem por zero. Considera-se, para tanto, as hipóteses estabelecidas anteriormente para o sinal de referência $r_{1}$. É importante mencionar que o detector de passagem por zero pode ser aplicado eficientemente mesmo quando a referência $r_{1}$ é obtida a partir da medição de uma variável elétrica, desde que algum processo de filtragem seja empregado para condicionar este sinal.

A implementação digital do detector de passagem por zero, considerando apenas as transições do semi-ciclo negativo para o positivo, é obtida através da seguinte equação:

$$
z_{c}(k)= \begin{cases}1, & \text { se } r_{1}(k-1)<0 \wedge r_{1}(k) \geq 0 \\ 0, & \text { caso contrário }\end{cases}
$$

O número de amostras entre dois cruzamento por zero consecutivos é dado por:

$$
m(k)=\left\{\begin{array}{cl}
m(k-1)+1, & \text { se } z_{c}(k)=0 \\
1, & \text { caso contrário }
\end{array}\right.
$$

A partir de $m$, pode-se estimar o número de amostras $\hat{n}$ entre os dois cruzamentos por zero do ciclo anterior:

$$
\hat{n}(k)=\left\{\begin{array}{cl}
m(k-1), & \text { se } z_{c}(k)=1 \\
\hat{n}(k-1), & \text { caso contrário }
\end{array}\right.
$$

Assumindo que $f_{s}$ não se altera quando $z_{c}=0$, i.e. entre dois cruzamentos por zero de $r_{1}$, o valor estimado de $f_{1}$ no

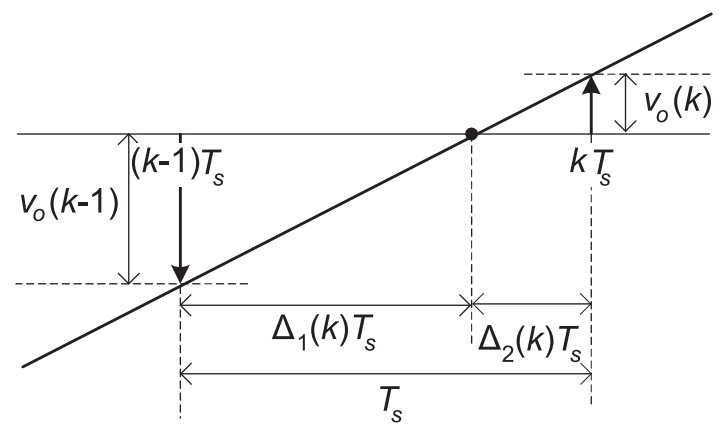

Fig. 3: Interpretação gráfica do método de interpolação utilizado para o detector de passagem por zero.

ciclo anterior da referência é dado por:

$$
\hat{f}_{1}(k)=\frac{f_{s}(k)}{\hat{n}(k)}
$$

Devido à representação de $\hat{n}$ apenas por valores inteiros, a eq. (15) pode resultar em erros significativos. De modo a reduzir este erro, é utilizado o algoritmo de interpolação cujo princípio é mostrado na Fig. 3. O método aproxima por uma reta a referência entre os instantes imediatamente anterior $(k-1) T_{s}$ e posterior $k T_{s}$ ao cruzamento por zero. Assim, é possível se aproximar o instante de passagem por zero $\left.(k-1) T_{s}+\Delta_{1}\right) T_{s}$. A estimativa corrigida de $\hat{n}$ é dada por:

$\hat{n}(k)=\left\{\begin{array}{c}m(k-1)+\Delta_{1}(k)+\bar{\Delta}_{2}(l)-\Delta_{3}(l), \text { se } z_{c}(k)=1 \\ \hat{n}(k-1), \text { caso contrário }\end{array}\right.$

onde $l=k-m(k-1)$

$$
\begin{gathered}
\Delta_{1}(k)=\frac{r_{1}(k-1)}{r_{1}(k-1)-r_{1}(k)} \\
\Delta_{2}(k)=1-\Delta_{1}(k) \\
\bar{\Delta}_{2}(l)=\Delta_{2}(l) \frac{f_{s}(l-1)}{f_{s}(l)}
\end{gathered}
$$

$$
\Delta_{3}(l)=\frac{f_{s}(l-1)}{f_{s}(l)}
$$

Comentário: O detector de passagem por zero é utilizado na implementação de todos os CRs neste artigo. Para implementar este algoritmo é importante destacar que:

1. Este algoritmo considera que $f_{s}$ pode ser alterada na passagem por zero;

2. As variáveis $\Delta_{1}(k), \Delta_{2}(k), \bar{\Delta}_{2}(l)$ e $\Delta_{3}(l)$ são calculadas apenas quando $z_{c}(k)=1$;

3. Para $f_{s}$ constante, $\bar{\Delta}_{2}(l)=\Delta_{2}(l)$ e $\Delta_{3}(l)=1$; 
4. Para as ações de controle calculadas na forma não preditiva, $\Delta_{3}(l)=1$.

\subsection{Controladores Repetitivos Modificados}

Os controladores comparados neste trabalho são descritos a seguir.

\subsubsection{Ajuste da Taxa de Amostragem (ATA)}

A Fig. 4(a) descreve este algoritmo, cuja igualdade descrita em (11) é obtida alterando $f_{s}$ e mantendo $n$ constante. O valor de $f_{s}$ é dado por:

$$
f_{s}(k+1)=\left\{\begin{array}{cc}
\frac{n^{*} f_{s}(k)}{\hat{n}(k)}, & \text { se } z_{c}(k)=1 \\
f_{s}(k), & \text { caso contrário }
\end{array}\right.
$$

onde $n^{*}=f_{s} / f_{1}^{*}$ é o valor de referência.

O desempenho deste CR depende fortemente do valor de $\hat{n}$. Consequentemente, é razoável admitir que existe uma notável melhoria de desempenho empregando-se (16) ao invés de (14).

\subsubsection{Truncamento do Número de Amostras de cada ciclo para o Inteiro Mais Próximo (TNAIMP)}

A Fig. 4(b) mostra o diagrama de blocos deste algoritmo. Este esquema de controle satisfaz a igualdade mostrada em (11) pela alteração do valor de $n$, mantendo $f_{s}$ constante. $\mathrm{O}$ valor de $n$ é obtido diretamente de (14), ou seja:

$$
n(k)=\hat{n}(k)
$$

É importante mencionar que se $n(k) \neq n(k-1)$, o tamanho dos buffers circulares utilizados para armazenar $u_{r p}$ e $e_{1}$ são ajustados. Quando $n(k)>n(k-1), n(k)-n(k-1)$ posições são adicionadas no final dos buffers circulares, cujos valores são inicializados em zero. Por outro lado, se $n(k)<n(k-$ $1), n(k-1)-n(k)$ posições são removidas do final destes buffers (Michels et al., 2009).

\subsubsection{Ajuste da Taxa de Amostragem Fictícia (ATAF)}

Este algoritmo é descrito na Fig. 4(c), cuja frequência de amostragem $f_{s}$ é constante. Para satisfazer (11), este algoritmo emprega uma frequência de amostragem fictícia $\tilde{f}_{s}$ ajustável para garantir o sincronismo entre os períodos do controlador e da referência. Para tanto, este algoritmo modifica $n$ para o valor igual ao inteiro mais próximo do número

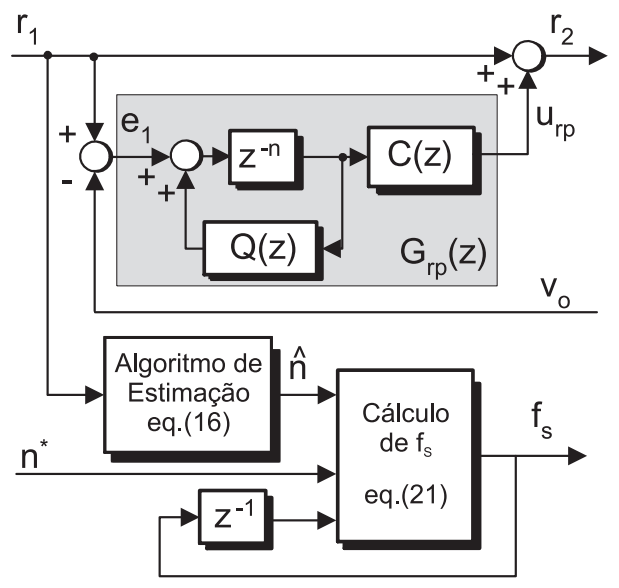

(a)

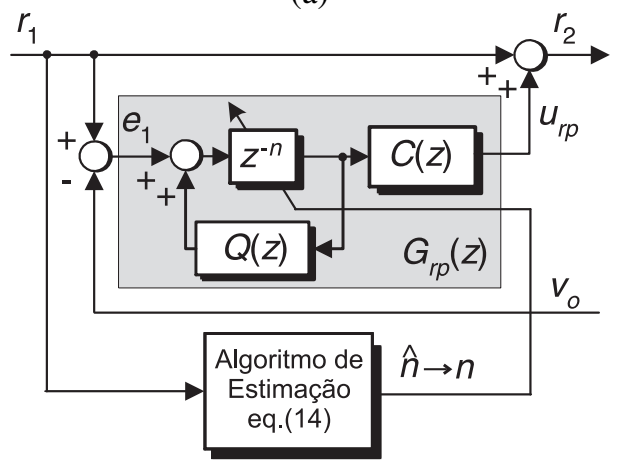

(b)

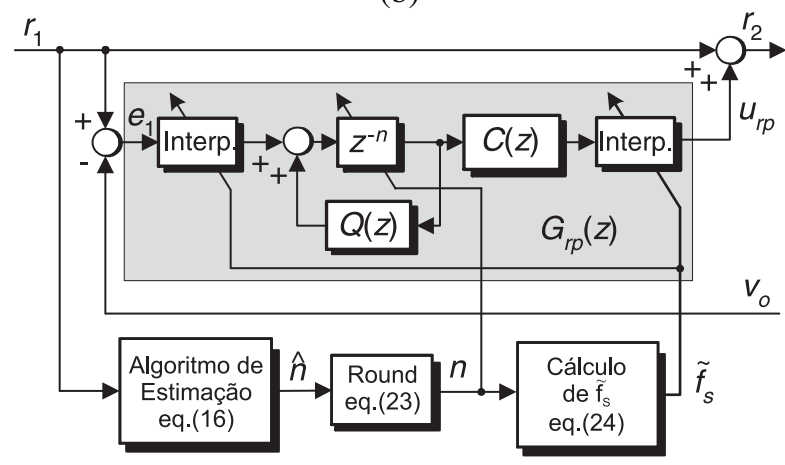

(c)

Fig. 4: CRs modificados. (a) ATA. (b) TNAIMP. (c) ATAF.

estimado de amostras por período (Cao and Ledwich, 2002). Os valores de $n$ e $\hat{f}_{s}$ são dados respectivamente por:

$$
\begin{gathered}
n(k)=\operatorname{round}(\hat{n}(k)) \\
\tilde{f}_{s}(k+1)=\left\{\begin{array}{cl}
\frac{\operatorname{round}(\hat{n}(k)) \tilde{f}_{s}(k)}{\hat{n}(k)}, & \text { se } z_{c}(k)=1 \\
\tilde{f}_{s}(k) & , \text { caso contrário }
\end{array}\right.
\end{gathered}
$$

O CR do tipo ATAF apresenta diferentes instantes de amostragem se comparado com o CR nominal, que possui uma taxa de amostragem fixa. Um algoritmo de interpolação gera 
amostras fictícias e sincronizadas com as do controlador. A ação de controle repetitiva $u_{r p}$ é calculada para estas amostras. Após, os valores resultantes são convertidos de modo a aproximar as amostragens nos instantes de tempo reais, utilizando para isto, o processo de interpolação inverso. O método de interpolação é o mesmo utilizado para determinar $\hat{n}$ na Sec. 3.1.

Pode-se observar que o algoritmo ATAF não possui problemas quanto a causalidade, uma vez que a ação de controle repetitiva faz uso das variáveis do ciclo anterior de $r_{1}$. É também importante mencionar que os buffers circulares devem ser ajustados conforme descrito na Sec. 3.2.2.

\section{RESULTADOS DA COMPARAÇÃO}

Com o objetivo de realizar uma análise comparativa dos três CRs modificados (ATA, TNAIMP and ATAF), apresentados na Sec. 3.2, foram construídos dois protótipos distintos. Os protótipos A e B são constituídos de um inversor PWM comutando com baixa e alta frequências de comutação, respectivamente. O objetivo de empregar duas plataforma experimentais distintas é a de contemplar aplicações em dois cenários distintos, de alta e de baixa potência, respectivamente. Os parâmetros destes protótipos são dados na Tabela 1, enquanto a Tabela 2 apresenta um maior detalhamento do hardware empregado nas implementações.

\subsection{Resposta em regime permanente}

A análise do comportamento do sistema em regime permanente avalia o desempenho do inversor com uma referência senoidal de frequência constante alimentando uma carga não-linear fixa. Para esta análise, a tensão de saída do inversor é medida e a distorção harmônica ou THD (do inglês Total Harmonic Distortion) é calculada quando o sistema atinge a operação nominal. A THD é utilizada como parâmetro de desempenho neste estudo.

Resultados de simulação para os CRs aplicados no Protótipo A são mostrados na Fig. 5, onde $f_{s}=6 \mathrm{kHz}$ e $f_{1}=$ $\{59,5 ; 60,5\} \mathrm{Hz}$. Estas simulações foram realizadas sem a inclusão da modulação por largura de pulso, a fim de ilustrar o comportamento dos algoritmos sem a interferência da implementação.

Os resultados experimentais para os CRs empregando os Protótipos A e B são mostrados nas Figs. 6 e 7, respectivamente. Para o Protótipo A foi escolhido $f_{s}=6 \mathrm{kHz}$ e $f_{1}=\{59,5 ; 60,5\} \mathrm{Hz}$, enquanto que para o Protótipo B, $f_{s}=18 \mathrm{kHz}$ e $f_{1}=\{58,59,60,61,62\} \mathrm{Hz}$.

A resposta em regime permanente do CR convencional é mostrada na Fig. 6(a) e 7(a). Este controlador resulta
Tabela 1: Parâmetros dos protótipos implementados.

\begin{tabular}{lccc}
\hline \hline & Parâmetro & Prot. A & Prot. B \\
\hline \hline \multirow{2}{*}{ Inversor } & Tensão do elo CC $\left(v_{c c}\right)$ & $200 \mathrm{~V}$ & $200 \mathrm{~V}$ \\
PWM & Potência nominal $\left(P_{o}\right)$ & $1 \mathrm{kVA}$ & $1 \mathrm{kVA}$ \\
& Freq. de amostragem $\left(f_{s}\right)$ & $6 \mathrm{kHz}$ & $18 \mathrm{kHz}$ \\
& Freq. de comutação $\left(f_{s w}\right)$ & $6 \mathrm{kHz}$ & $18 \mathrm{kHz}$ \\
\hline \multirow{2}{*}{ Referência } & Amplitude $\left(v_{r e f}\right)$ & $110 V_{r m s}$ & $110 V_{r m s}$ \\
& Frequência base $\left(f_{b a s e}\right)$ & $60 \mathrm{~Hz}$ & $60 \mathrm{~Hz}$ \\
\hline \multirow{4}{*}{ Filtro } & Indut. do filtro $(L)$ & $1 \mathrm{mH}$ & $0,4 \mathrm{mH}$ \\
$L C$ & Resist. do indutor $\left(r_{L}\right)$ & $0,1 \Omega$ & $0,1 \Omega$ \\
& Capacit. do filtro $(C)$ & $35 \mu \mathrm{F}$ & $25 \mu \mathrm{F}$ \\
\multirow{2}{*}{ Controle } & Resist. do capacitor $\left(r_{C}\right)$ & $0,05 \Omega$ & $0,05 \Omega$ \\
PD-FF & Ganho $k_{1}$ & $-0,168$ & 0,073 \\
\hline \multirow{4}{*}{ CR } & Ganho $k_{2}$ & $-0,014$ & $-0,337$ \\
\hline \hline \multirow{2}{*}{ Carga } & Amortecimento $q_{r}$ & 0,99 & 0,99 \\
não-linear & Ganho $c_{r}$ & 0,10 & 0,10 \\
& Compensação de fase $(d)$ & 2 & 2 \\
& Número de amostras $(n)$ & 100 & 300 \\
\hline \hline
\end{tabular}

Tabela 2: Detalhes construtivos dos protótipos.

\begin{tabular}{lcc}
\hline \hline Característica & Protótipo A & Protótipo B \\
\hline \hline Padrão PWM & três níveis & três níveis \\
Tipo de pulso PWM & centralizado & centralizado \\
Chave semicondutora & IGBT & IGBT \\
Núcleo do indutor & ferro-silício & pó de ferro \\
Capacitor & polipropileno & polipropileno \\
Plataforma & PC/DAQ & DSP TMS28x \\
Formato das variáveis & ponto flutuante & ponto fixo \\
Tamanho das variáveis & 32 bits & 32 bits \\
Conversor A/D & 12 bits & 12 bits \\
Timers do PWM & 16 bits & 16 bits \\
\hline \hline
\end{tabular}



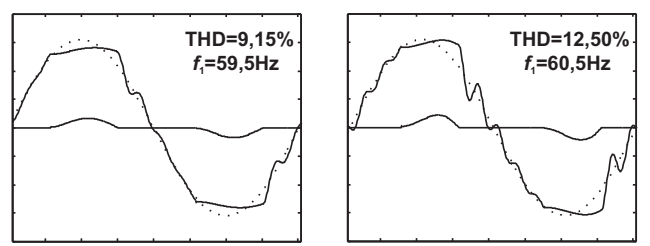

(a)
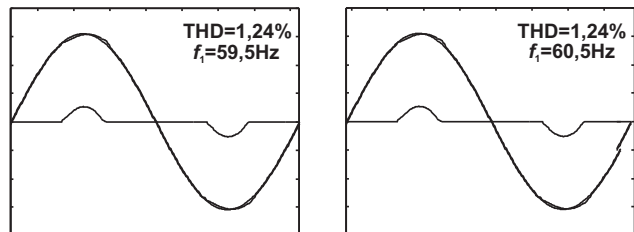

(b)
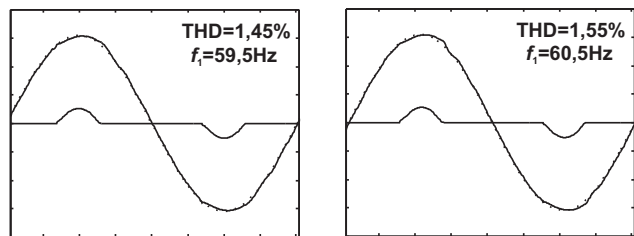

(c)
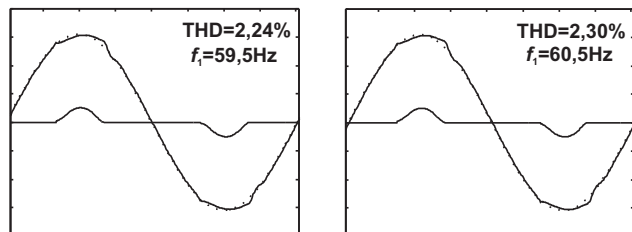

(d)

Fig. 5: Resultados de simulação em regime permanente para o Protótipo A (50V/div; 50A/div). (a) CR convencional. (b) ATA. (c) TNAIMP. (d) ATAF.

em valores elevados de THD quando os distúrbios cíclicos não estão sincronizados com o período da ação repetitiva $\left(f_{1} \neq f_{1}^{*}\right)$, como previsto na simulação mostrada na Fig. 5(a). Observa-se que existem pequenas diferenças entre as formas de onda obtidas em simulação e experimentalmente. Estas se devem à existência de amortecimentos na planta e de dinâmicas não-modeladas que não foram incluídos no modelo de simulação.

Por outro lado, os três algoritmos repetitivos descritos na Seção 3.2 apresentam uma reduzida THD na mesma situação de não sincronização. $\mathrm{O}$ bom desempenho destes algoritmos pode ser verificada nos resultados de simulação, mostrados na Fig. 5(b-d), e nos resultados experimentais, mostrados nas Figs. 6(b-d) e 7(b-d). Observa-se que os resultados de experimentais para o Protótipo A possuem uma forma de onda que visualmente assemelha-se aos das respectivas simulações, mas cujos valores de THD não seguem a mesma tendência dos resultados de simulação. Este comportamento é devido à interação das harmôncias de modulação por largura de pulso com àquelas associadas ao controlador. Como
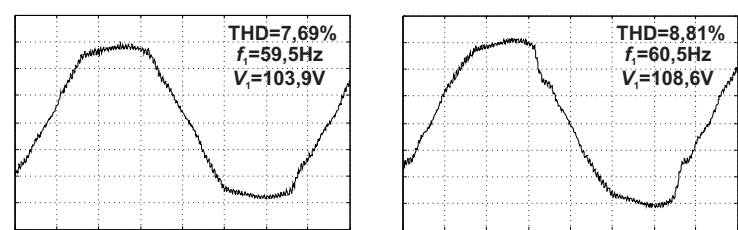

(a)
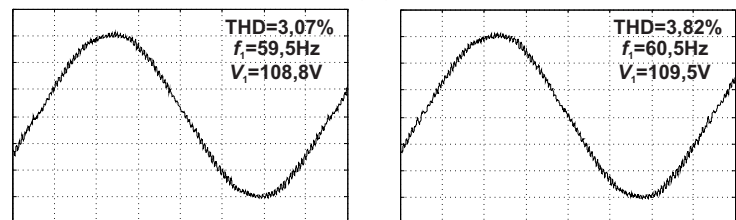

(b)
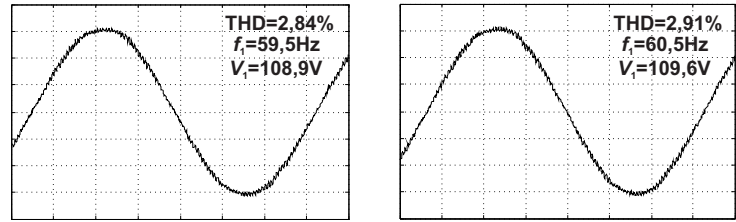

(c)
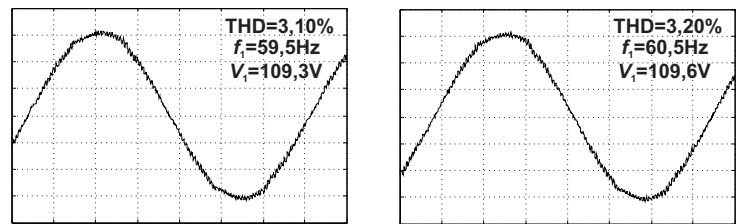

(d)

Fig. 6: Resultados experimentais em regime permanente para o Protótipo A (50V/div; $2 \mathrm{~ms} / \mathrm{div})$. (a) CR convencional. (b) ATA. (c) TNAIMP. (d) ATAF.

ambos os grupos de harmônicas possuem a mesma ordem de grandeza (em torno de $2 \%$ ), as interações construtivas e destrutivas entre as mesmas, associadas ao efeito não-linear da carga, influenciam na THD resultante.

As Figs. 6(b) e 7(b) mostram o desempenho do algoritmo ATA em regime permanente. Pode-se verificar que este algoritmo resulta num bom desempenho, apresentado uma reduzida taxa de distorção harmônica para toda a faixa de frequência analisada. Este algoritmo comporta-se de forma similar a um controlador repetitivo convencional quando seu período torna-se sincronizado com o período do sinal de referência.

As formas de onda para o algoritmo TNAIMP são mostradas nas Figs. 6(c) e 7(c). Pode-se verificar que este algoritmo resulta em bom desempenho em regime permanente. Contudo, apesar de não ser possível visualizar no gráfico devido ao intervalo de visualização reduzido, constata-se a presença de oscilações de pequena amplitude na tensão de saída. Isto se deve ao truncamento cíclico no valor de $n$ quando $f_{1} \neq f_{1}^{*}$. As oscilaçoes geram componentes subharmônicas 
de pequena amplitude, cuja distribuição espectral depende do ponto de operação ${ }^{2}$.

Uma análise do impacto das oscilações na THD da tensão de saída para o algoritmo TNAIMP foi realizada através de simulação. Para tanto, realizou-se um conjunto de simulações variando-se a $f_{1}$ em $0,01 \mathrm{~Hz}$ na faixa entre $58 \mathrm{~Hz}$ e 62 Hz. A partir destes resultados foram determinados os valores da THD da tensão de saída para cada ciclo, onde observou-se que esta apresenta pequenas variações ciclo-a-ciclo devido as oscilações. As maiores variações da THD para os Protótipos A e B foram de $0,3 \%$ e $0,1 \%$, respectivamente.

Observa-se que amplitude da oscilação aumenta a medida que $n^{*}$ diminui devido ao aumento do percentual de responsabilidade do truncamento. Contudo, esta amplitude não pode ser obtida através de uma relação matemática, pois depende também dos parâmetros do filtro de saída, dos controlador empregados e da carga. Por este motivo, para garantir um bom funcionamento, recomenda-se que o algoritmo TNAIMP seja analisado por simulação para valores de $n<100$, para verificar se a amplitude destas oscilações são suficientemente reduzidas.

Os resultados para o algoritmo ATAF são mostrados nas Figs. 6(d) e 7(d). Apesar dos resultados apresentados para este algoritmo resultarem num desempenho em regime permanente semelhante ao ATA e TNAIMP, é importante destacar que este algoritmo tende a apresentar uma THD mais elevada se as harmônicas forem de ordem elevada. Esta característica se deve à baixa atenuação do $\mathrm{CR}$ nas altas frequências devido às interpolações efetuadas. A operação de interpolação resulta num atraso de fase nulo, mas possui uma característica passa-baixa. Esta característica é análoga à do filtro $Q\left(z^{-1}\right)$ utilizando em CRs convencionais. Como resultado, este algoritmo apresenta um bom desempenho quando a frequência de amostragem é muito maior que a frequência da referência e das harmônicas da carga. Além disso, é o algoritmo mais adequado para aplicações onde $f_{1}^{*}$ pode possuir uma ampla faixa de valores.

\subsection{Resposta transitória}

A análise da resposta transitória avalia o desempenho do inversor alimentando uma carga não-linear fixa com uma referência senoidal durante as variações de frequência. A frequência da referência é modificada linearmente a uma taxa de $1 \mathrm{~Hz} / \mathrm{s}$, tal como assumido na Sec. 3.

A análise da resposta transitória foi efetuada através da medida do erro de rastreamento $e_{1}$ durante as variações da frequência base $f_{1}$ da referência. A amplitude do erro $e_{1}$ é a

\footnotetext{
${ }^{2}$ Os valores de THD apresentados nas Figs. 6(c) e 7(c) foram obtidos a partir de uma média de vários períodos.
}

medida utilizada para a comparação de desempenho. Quanto menor for o valor do erro, melhor é o desempenho transitório do CR.

Resultados experimentais da resposta transitória para o Protótipo A são mostrados na Fig. 8. O sinal de referência $r_{1}$ tem sua frequência base alterada linearmente entre $t_{1}$ e $t_{2}$, desde $f_{1}^{*}=60 \mathrm{~Hz}$ até $f_{1}=60,5 \mathrm{~Hz}$ (coluna da esquerda) e de $f_{1}^{*}=60 \mathrm{~Hz}$ para $f_{1}=59,5 \mathrm{~Hz}$ (coluna da direita). A resposta transitória para o Protótipo B é mostrado na Fig 9. Neste caso, a frequência da referência $f_{1}$ é modificada linearmente de $58 \mathrm{~Hz}$ para $62 \mathrm{~Hz}$ (coluna da esquerda) e de 62 $\mathrm{Hz}$ para $58 \mathrm{~Hz}$ (coluna da direita).

As respostas transitórias do CR convencional são mostradas nas Figs. 8(a) e 9(a). Pode-se observar que o erro é reduzido para frequências $f_{1}$ próximas de $f_{1}^{*}=60 \mathrm{~Hz}$, mas aumenta significativamente a medida que $f_{1}$ é alterada. Pode-se verificar a partir das Figs. 8(a) e 9(a) que o erro é menor quando $f_{1}<60 \mathrm{~Hz}$, se comparado com o caso onde $f_{1}>60 \mathrm{~Hz}$.

As Figs. 8(b-d) e Fig. 9(b-d) mostram que os algoritmos ATA, TNTSI e ASFR possuem desempenho transitório semelhantes. Em todos os casos, não se observam modificações significativas na magnitude do erro durante a variação da frequência da referência. Do ponto de vista de comparação, conclui-se que todos os algoritmos repetitivos apresentados na Sec. 3 possuem um bom comportamento transitório nas condições assumidas para variação do sinal de referência.

\subsection{Custo computacional}

A Tabela 3 mostra os tempos de execução do código utilizado para a implementação dos algoritmos repetitivos convencional, ATA, TNAIMP e ATAF. Estes tempos de execução foram obtidos para o Protótipo $\mathrm{B}$, cujas leis de controle foram implementadas no DSP TMS320F2812.

Os resultados mostram que os algoritmos ATA e TNAIMP necessitam de apenas $4,6 \%$ de tempo de processamento adicional com relação ao CR convencional. Por outro lado, o algoritmo ATAF necessita de 119,5\% de tempo de processamento adicional em relação ao mesmo algoritmo. Quando todas as rotinas de controle são consideradas, o tempo de execução adicional do algoritmo ATAF é de $22,2 \%$.

\section{CONCLUSÃo}

Este artigo apresenta uma análise comparativa de CRs modificados aplicados a inversores de tensão PWM para o rastreamento de sinais de referência de frequência variável. Os três algoritmos foram analisados experimentalmente em condições idênticas, empregando dois protótipos com frequências de amostragem distintas $(N=100$ e $N=300)$. 


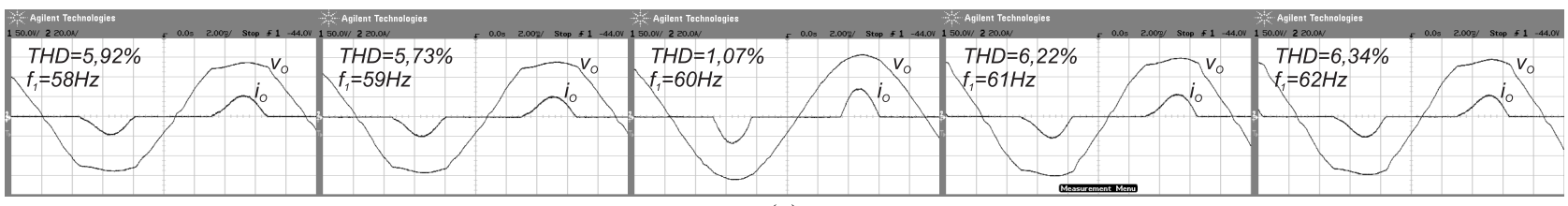

(a)

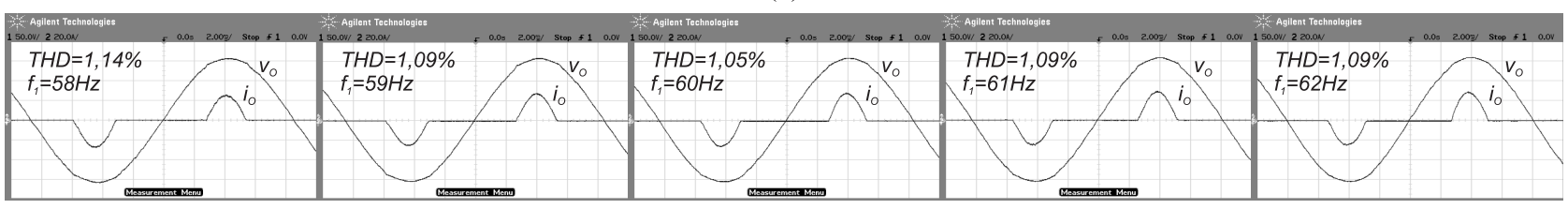

(b)

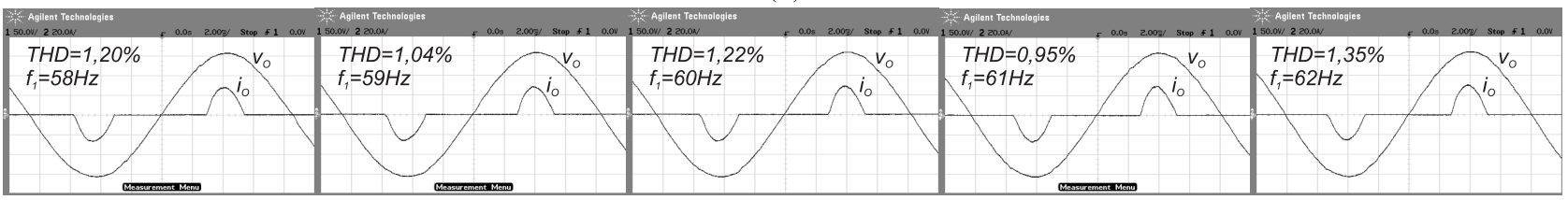

(c)

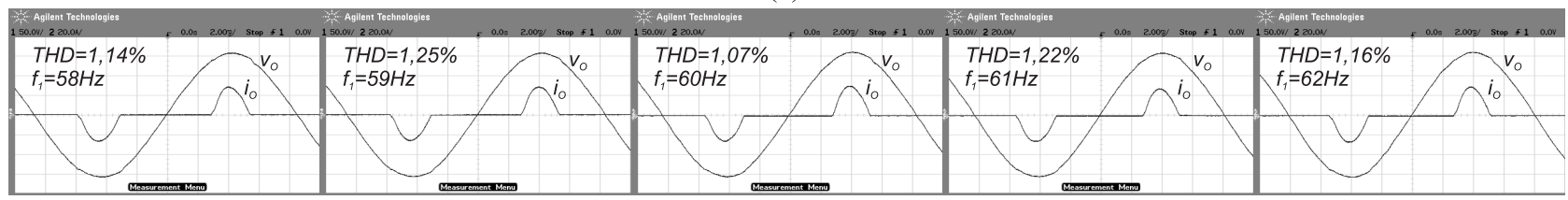

(d)

Fig. 7: Resultados experimentais em regime permanente para o Protótipo B (50V/div; 20A/div; 2ms/div). (a) CR convencional. (b) ATA. (c) TNAIMP. (d) ATAF.

Tabela 3: Tempo de execução dos algoritmos de controle no Protótipo B.

\begin{tabular}{lcc}
\hline \hline \multicolumn{1}{c}{ Tipo de CR } & Rotina do CR & Todas as rotinas de controle \\
\hline \hline Convencional & $2,61 \mu \mathrm{s}$ & $14,4 \mu \mathrm{s}$ \\
ATA & $2,73 \mu \mathrm{s}$ & $14,8 \mu \mathrm{s}$ \\
TNAIMP & $2,71 \mu \mathrm{s}$ & $14,8 \mu \mathrm{s}$ \\
ATAF & $5,73 \mu \mathrm{s}$ & $17,6 \mu \mathrm{s}$ \\
\hline \hline
\end{tabular}

Os três algoritmos analisados reduzem significativamente a taxa de distorção harmônica da tensão de saída, em regime permanente, em comparação ao $\mathrm{CR}$ convencional. Analisando-se todos os testes realizados, observa-se que a mínima redução na taxa de distorção harmônica total é de $4,5 \%$. Constata-se, também, que não há significativa diferença de desempenho em regime permanente entre os três algoritmos.

O desempenho transitório dos algoritmos também foi avaliado para uma taxa de variação da frequência do sinal de referência de $1 \mathrm{~Hz} / \mathrm{s}$. Observa-se que o erro nos transitórios aumenta em mais de $15 \mathrm{~V}$ no CR convencional, enquanto mantém-se aproximadamente constante nos três CRs modifi- cados. Como a distorção harmônica é uma função do erro na tensão de saída, conclui-se que os CRs modificados não apresentam significativas distorções na tensão de saída durante as variações da frequência da referência. Tal como no caso anterior, observa-se que os três CRs modificados apresentam comportamento similares entre si.

Portanto, para os casos consideradas neste estudo, os três CRs modificados analisados possuem desempenho similares entre si. Contudo, observa-se que o algoritmo ATAF tem a desvantagem de demandar um tempo computacional duas vezes superior aos algoritmos ATA e TNAIMP.

\section{AGRADECIMENTOS}

Os autores gostariam de agradecer ao CNPq (Edital MCT/CNPq 14/2009 - Proc. 478199/2009-0), à CAPES e à FAPERGS pelo apoio financeiro ao desenvolvimento deste estudo.

\section{REFERÊNCIAS}

Bottern, F. and Pinheiro, H. (2005). Synchronous frame halfperiod repetitive controller for three-phase ups, Anais do VIII Congresso Brasileiro de Eletrnica de Potncia, 

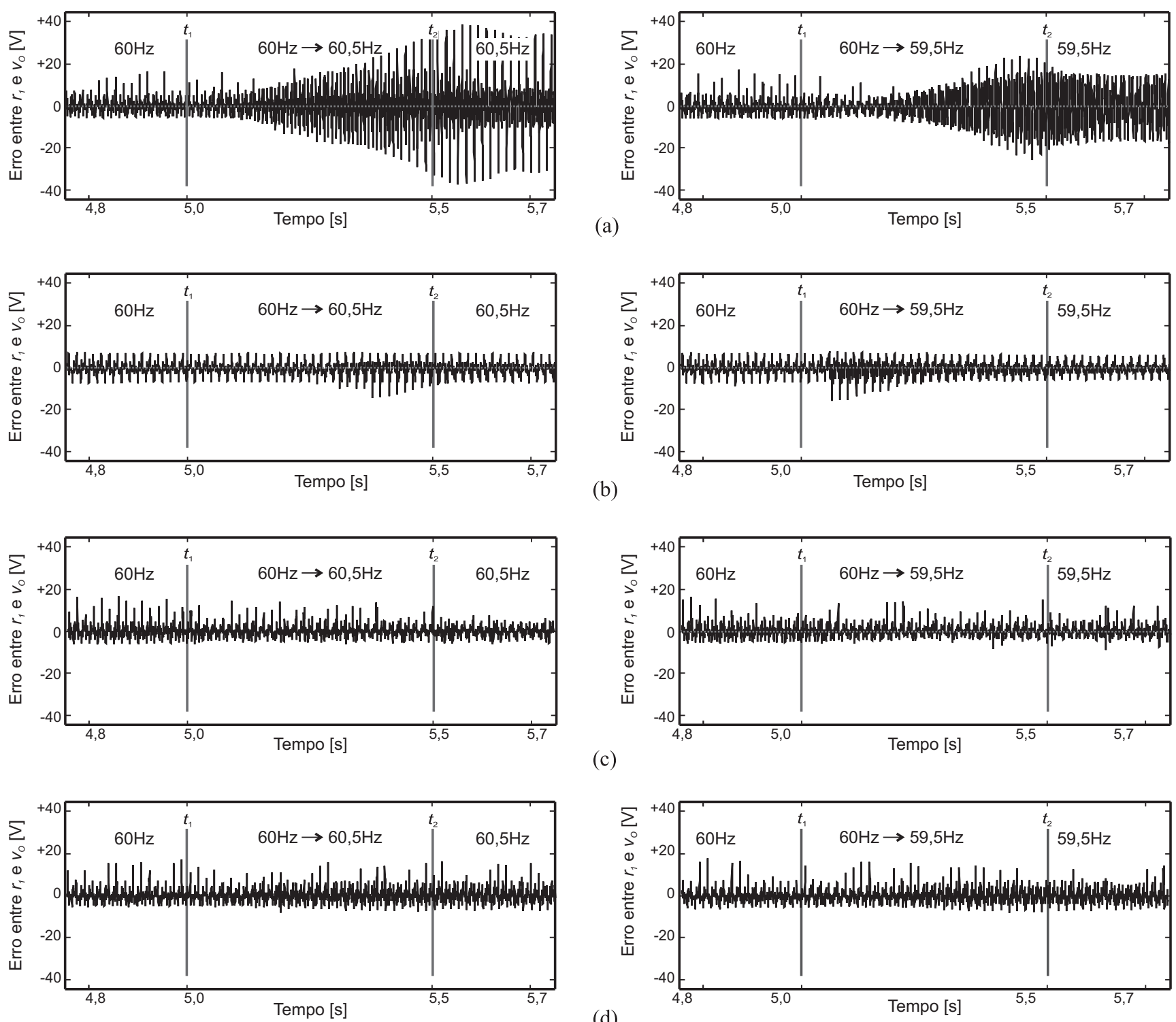

(c)

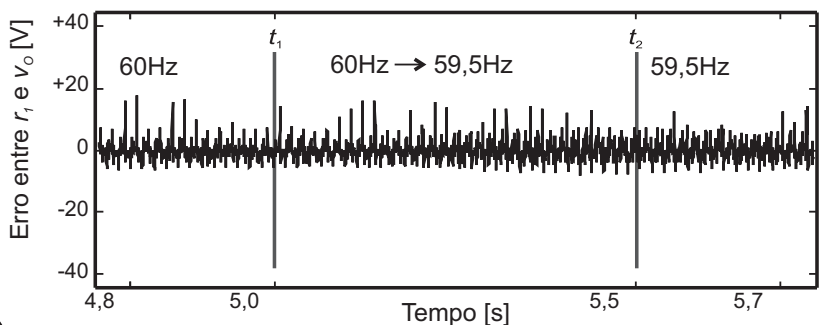

Fig. 8: Resultados experimentais do comportamento transitório para o Protótipo A. (a) CR convencional. (b) ATA. (c) TNAIMP. (d) ATAF.

pp. $444-450$.

Cao, Z. and Ledwich, G. F. (2002). Adaptive repetitive control to track variable periodic signals with fixed sampling rate, IEEE Trans. Contr. Syst. Technol. 7(3): 378384.

Contreras, S. A. D., Cortizo, P. C., Mendes, M. A. S. and Filho, R. M. S. (2010). Analysis of digital controllers for equilibrated current distribution in an interleaved inverter, Conf. Records of 9th IEEE/IAS International Conference on Industry Applications (INDUSCON), pp. 1-6. de Melo, P., Gules, R., Annunziato, R. C. and Romaneli, E. F. R. (2010). Development and implementation of a three phase active filter, Conf. Records of 9th IEEE/IAS International Conference on Industry Applications (INDUSCON), pp. 1-6.

Escobar, G., Leyva-Ramos, J., Martinez, P. R. and Valdez, A. A. (2005). A repetitive-based controller for the boost converter to compensate the harmonic distortion of the output voltage, IEEE Trans. Contr. Syst. Technol. 13(3): $500-508$.

Escobar, G., Valdez, A. A., Leyva-Ramos, J. and Mattavelli, 


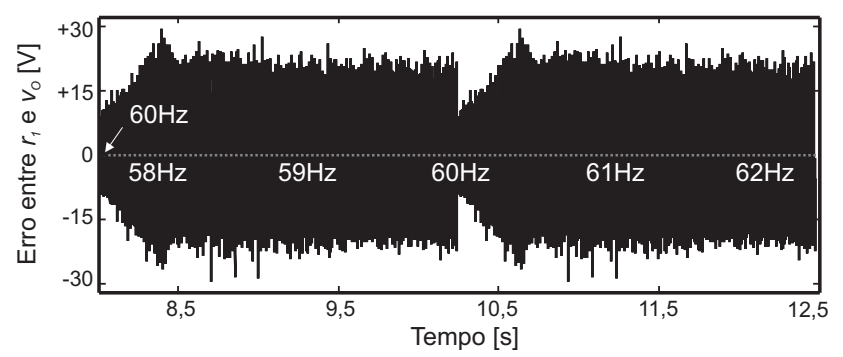

(a)

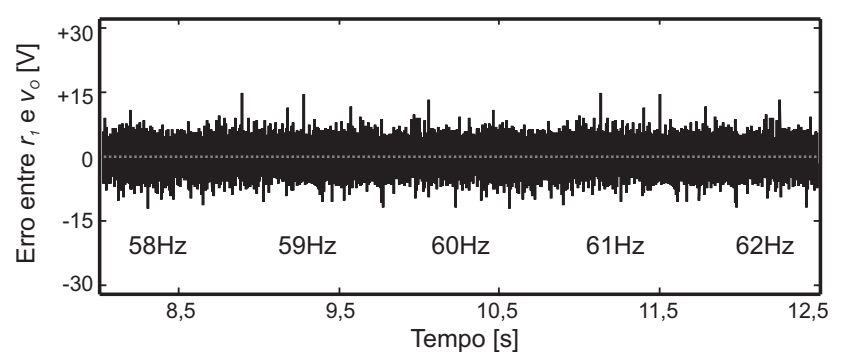

(b)

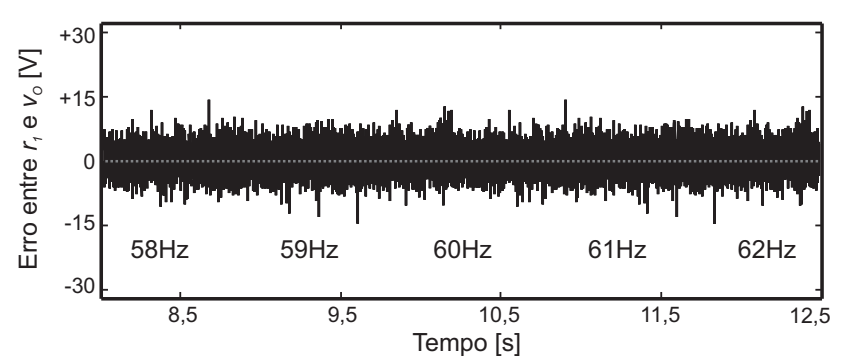

(c)
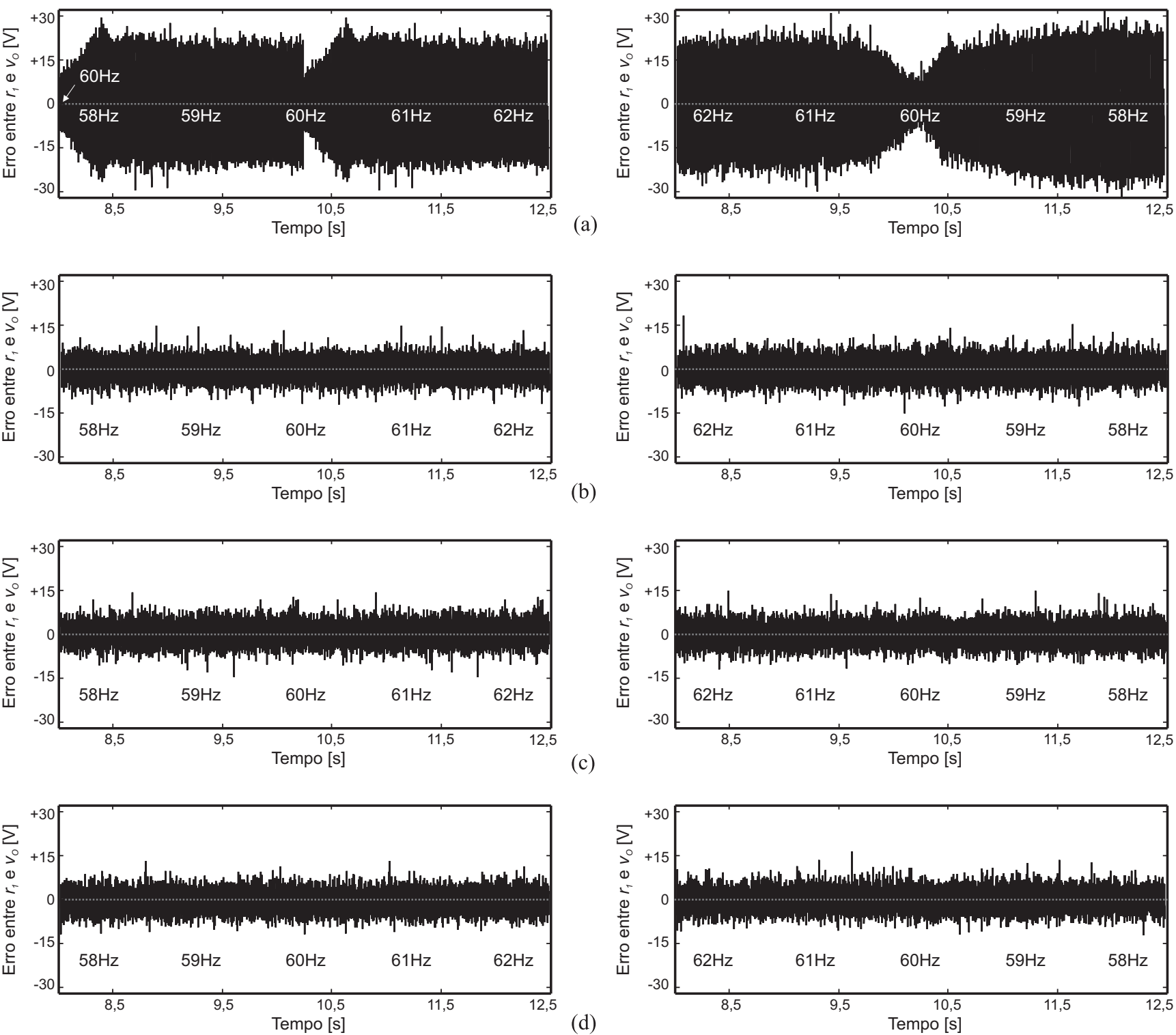

(d)

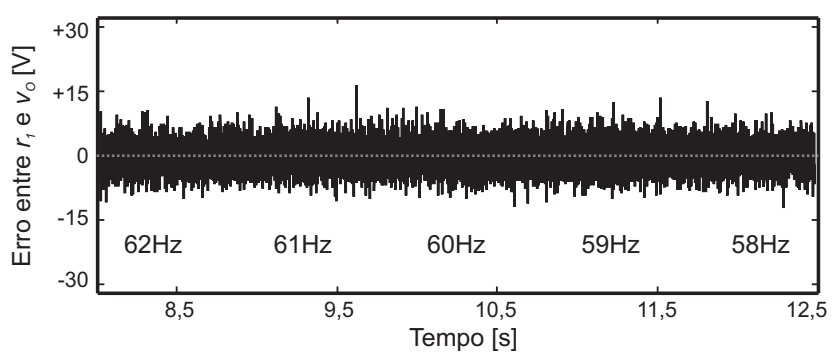

Fig. 9: Resultados experimentais do comportamento transitório para o Protótipo B. (a) CR convencional. (b) ATA. (c) TNAIMP. (d) ATAF.

P. (2007). Repetitive-based controller for a UPS inverter to compensate unbalance and harmonic distortion, IEEE Trans. Ind. Electron. 54(1): 504-510.

Flores, J. V., Pereira, L. F. A., Jr., J. M. G. S., Bonan, G. and Coutinho, D. F. (2010). LMI-based design of robust repetitive controllers for UPS systems, Anais do XVIII Congresso Brasileiro de Automtica, pp. 4104-4109.

Francis, B. A. and Wonham, W. M. (1975). The internal model principle for linear multivariable regulators, Appl. Math. Optimization, 2(1): 170-194.
Fujimoto, H. (2009). RRO compensation of hard disk drives with multirate repetitive perfect tracking control, IEEE Trans. Ind. Electron. 56(10): 3825 -3831.

García-Cerrada, A., Pinzón-Ardila, O., Feliu-Batlle, V., Roncero-Sánchez, P. and García-Gonzalez, P. (2007). Application of a repetitive controller for a threephase active power filter, IEEE Trans. Power Electron. 22(1): 237-246.

Grino, R., Cardoner, R., Costa-Castello, R. and Fossas, E. (2007). Digital repetitive control of a three-phase four- 
wire shunt active filter, Industrial Electronics, IEEE Transactions on 54(3): $1495-1503$.

Gupta, M. and Lee, J. H. (2006). Period-robust repetitive model predictive control, Journal of Process Control 16(6): 545-555.

Hara, S., Yamamoto, Y., Omata, T. and Nakano, M. (1988). Repetitive control system: A new type servo system for periodic exogenous signals, IEEE Trans. Autom. Contr. 33(7): 659-668.

Hillerstrom, G. (1996). Adaptive suppression of vibrations A repetitive control approach, IEEE Trans. Contr. Syst. Technol. 4(1): $72-78$.

Hornik, T. and Zong, Q.-C. (2010). $H^{\infty}$ repetitive voltage control of grid-connected inverters with a frequency adaptive mechanism, IET Power Electron. 3(6): 925935.

IEC 62040-3 (1999). Uninterruptible Power Systems (UPS) Part 3: Method of specifying the performance and test requirements, International Electrotechnical Commission.

Jung, S.-L., Huang, H.-S. and Tzou, Y.-Y. (1998). A threephase PWM AC-DC converter with low swithcing frequency and high power factor using DSP-based repetitive control technique, Conference Records of 29th IEEE PESC, Fukuoka, Japan, pp. 517-523.

Kempf, C., Messner, W. and Tomizuka, M. (1993). Comparison of four discrete-time repetitive control algorithm, IEEE Contr. Syst. Mag. 13(6): 48-54.

Manayathara, T., Tsu-Chin, T. and Bentsman, J. (1996). Rejection of unknown periodic load disturbances in continuous steel casting process using learning repetitive control approach, IEEE Trans. Contr. Syst. Technol. 4(3): $259-265$.

Marafo, F. P., Mattavelli, P., Buso, S. and Deckmann, S. M. (2004). Repetitive-based control for selective active filters using discrete cosine transform, SOBRAEP Eletrnica de potncia 9 (1): 26-36.

Mastromauro, R. A., Liserre, M., Kerekes, T. and Dell'Aquila, A. (2009). A single-phase voltagecontrolled grid-connected photovoltaic system with power quality conditioner functionality, IEEE Trans. Ind. Electron. 56(11): 4436-4444.

Mattavelli, P. and Marafao, F. P. (2004). Repetitive-based control for selective harmonic compensation in active power filters, IEEE Trans. Ind. Electron. 51(5): 10181024.
Michels, L. and Gründling, H. A. (2005). Procedimento de projeto de controladores repetitivos para o estgio de sada de fontes ininterruptas de energia, SOBRAEP Eletrnica de Potncia 10(1): 39-50.

Michels, L., Pinheiro, H. and Gründling, H. A. (2004). Design of plug-in repetitive controllers for single-phase PWM inverters, Conference Records of the 39th IAS Annual Meeting, Seattle, EUA, pp. 163-170.

Michels, L., Rech, C., Almeida, R. S., Soares, C. E. and Mezaroba, M. (2010). Tcnicas anti-windup para controladores repetitivos empregados em inversores PWM, Anais do XVIII Congresso Brasileiro de Automtica, pp. $845-851$.

Michels, L., Stefanello, M. and Gründling, H. A. (2009). Controlador repetitivo para inversores PWM com referência de freqüência variável, Controle \& Automação 20(3): 394-404.

Montagner, V. F. and Peres, P. L. D. (2007). Design of robust repetitive controllers based on convex optimization with an application to power electronics, Anais do IX Congresso Brasileiro de Eletrnica de Potncia, pp. 22 25.

Olm, J. M., Ramos, G. A., Castello-Castelló, R. and Cardoner, R. (2010). Odd-harmonic repetitive control of an active filter under varying network frequency: Control design and stability analysis, Proceedings of the American Control Conference (ACC), 2010 IEEE, IEEE, pp. 1749-1754.

Olm, J. M., Ramos, G. A., Costa-Castelló, R. and Cardoner, R. (2010). Odd-harmonic repetitive control of an active filter under varying network frequency: Control design and stability analysis, Proceedings of the 2010 American Control Conference, 2010 IEEE, IEEE, Baltimore, MD, USA, pp. 1749-1754.

Olm, J., Ramos, G., and Costa-Castelló, R. (2010). Adaptive compensation strategy for the tracking/rejection of signals with time-varying frequency in digital repetitive control systems, Journal of Process Control 20(4): 551-558.

Pipeleers, G., Demeulenaere, B., Schutter, J. D. and Swevers, J. (2008). Robust high-order repetitive control: Optimal performance trade-offs, Automatica 44(10): 26282634

Rech, C., Michels, L. and Pinheiro, J. R. (2010). Reinicializao de controladores repetitivos em inversores pwm sob distrbios no-peridicos: Anlise e projeto, SOBRAEP Eletrnica de potncia 15(1): 11-20. 
Stefanello, M. and Grndling, H. A. (2007). An adaptive pd controller with odd-harmonic repetitive compensation for ups applications, Anais do IX Congresso Brasileiro de Eletrnica de Potncia, pp. 444-450.

Steinbuch, M. (2002). Repetitive control for systems with uncertain period-time, Automatica 38(12): 2103-2109.

Tomizuka, M., Tsao, T.-C. and Chew, K.-K. (1989). Analysis and synthesis of discrete-time repetitive controllers, Trans. ASME: J. Dyn. Syst., Meas., Contr. 111: 353358.

Tzou, Y.-Y., Ou, R.-S., Jung, S.-L. and Chang, M.-Y. (1997). High-performance programmable AC power source with low harmonic distortion using DSP-based repetitive control technique, IEEE Trans. Power Electron. 12(4): 715-725.

Wang, Y., Wang, D., Zhang, B. and Zhou, K. (2007). Fractional delay based repetitive control with application to PWM DC/AC converters, Conference Record of the IEEE 22nd ISIC, Singapore, pp. 928-933.

Wu, X., Panda, S. and Xu, J. (2010). Design of a plugin repetitive control scheme for eliminating supply-side current harmonics of three-phase PWM boost rectifiers under generalized supply voltage conditions, IEEE Trans. Power Electron. 25(7): 1800-1810.

Zhou, K., Low, K.-S., Wang, D., Luo, F.-L., Zhang, B. and Wang, Y. (2006). Zero-phase odd-harmonic repetitive controller for a single-phase PWM inverter, IEEE Trans. Power Electron. 21(1): 193-201. 